\title{
Probing matter with relativistic electron beams
}

Intense ultrashort electron beams can peer into the changes in the microscopic features of materials taking place on femtosecond time scales. Using particle acceleratorinspired technologies, electrons can now be rapidly accelerated to relativistic energies; however, despite these advances the spatial resolution of ultrafast electronbased techniques is still low compared with traditional electron microscopes.

$\mathrm{Fu}-\mathrm{Hao} \mathrm{Ji}$ and colleagues used a high-brightness highrepetition rate electron scattering

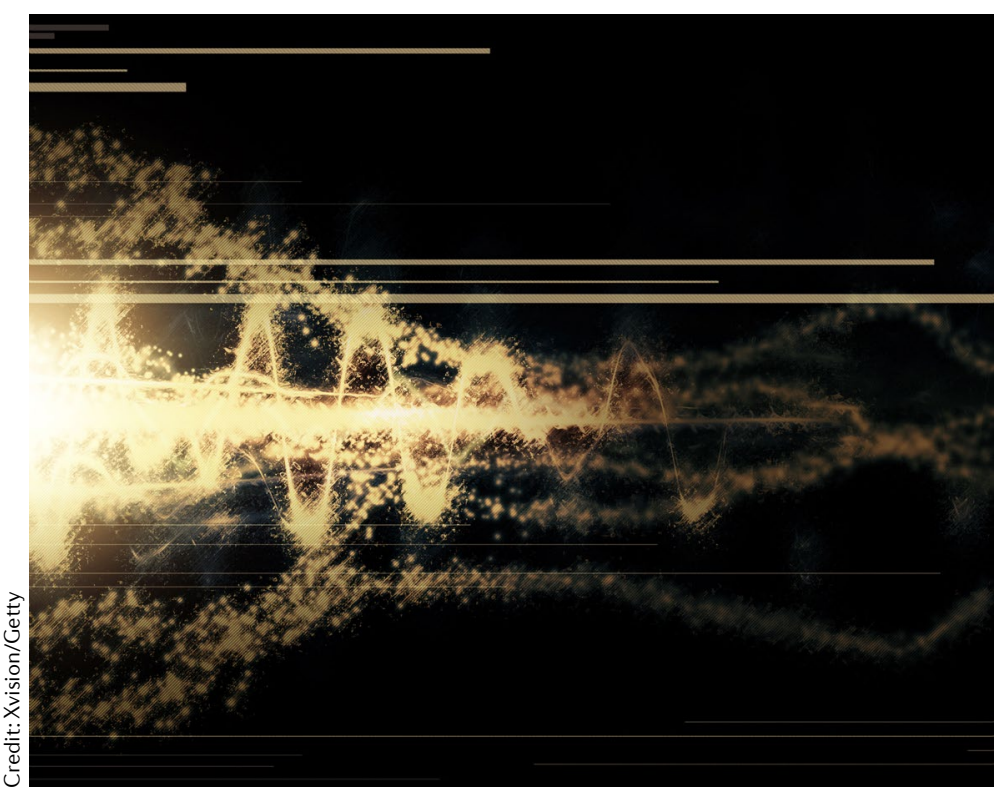

source - the Advanced

Photoinjector Experiment recently developed at the Lawrence Berkeley National Laboratory - to generate ultrafast relativistic electron beams two orders of magnitude brighter than in previous studies. With this high-quality beam the authors demonstrated ultrafast electron diffraction with nanoscale spatial resolution and the potential for scanning transmission electron microscopy. Although the resolution is still not at the level of traditional electron microscopes, it is smaller than the grain size in most materials and hence sufficient to probe structural variations. Relativistic ultrafast electron diffraction with its femtosecond timescale and nanometre resolution holds promise for the study of real-time energy transfer in materials, which underlies many interesting physical phenomena.

Anastasiia Novikova

ORIGINAL ARTICLE Ji, F. et al. Ultrafast relativistic electron nanoprobes. Commun. Phys. https://doi. org/10.1038/s42005-019-0154-4 (2019). 